\title{
AuCu II, STRUCTURE MODULÉE IRRATIONNELLE, PROTOTYPE DES ANTIPHASES PÉRIODIQUES
}

\author{
M. GUYMONT et D. GRATIAS (*)
}

Laboratoire de Cristallographie et Physique des Matériaux

Bât. 490, Université de Paris XI, 91405 Orsay Cedex, France

(*) Laboratoire de Métallurgie Structurale des Alliages Ordonnés, ENSCP

11, rue Pierre-et-Marie-Curie, 75231 Paris Cedex, France

(Reçu le 21 juillet 1978, accepté le 3 octobre 1978)

\begin{abstract}
Résumé. - Nous avons observé par diffraction électronique à partir d'échantillons massifs de $\mathrm{AuCu}$ II à la composition 50 at. \% Au la structure Johansson et Linde exacte (demi-période d'antiphase $M=5$ ). L'examen au microscope électronique montre qu'il s'agit d'une véritable antiphase périodique (modèle Jehanno-Pério) par opposition à $\mathrm{Ag}_{3} \mathrm{Mg}$ ordonné qui constitue une structure à longue période rationnelle (modèle Fujiwara). Ceci est confirmé à la composition 55 at. \% $\mathrm{Au}$ où on observe le chevauchement des réflexions d'antiphase provenant de deux mailles réciproques consécutives.
\end{abstract}

Abstract. - Exact Johansson-Linde structure is observed by electron diffraction from 50 at. \% $\mathrm{AuCu}$ II bulk samples (antiphase half period $M=5$ ). Nevertheless, electron micrography shows a periodic antiphase structure (contrary to ordered $\mathrm{Ag}_{3} \mathrm{Mg}$ ) as confirmed on a 55 at. \% Au sample which shows overlapping without superposition of antiphase reflections coming from neighbouring reciprocal cells.

1. Introduction. - La structure à antiphases périodiques (APP) $\mathrm{AuCu}$ II, stable entre environ 37 et 65 at. \% $\mathrm{Au}$, a été découverte par diffraction des rayons X sur poudre par Johansson et Linde [1]. Ceci constituait la première structure «irrationnelle », la période $2 M$ n'étant pas un nombre simple de mailles. Par la suite, grâce à l'étude par microscopie électronique de lames évaporées [2,3] ou par diffraction des rayons $X$ sur poudres $[4,5]$ ou sur monocristaux $[5,6]$, on a pu préciser le modèle. En particulier :

1) A la stœchiométrie, la précision des mesures $(M=5,13)$ exclut la valeur entière $M=5$.

2) Lorsqu'on s'écarte de la stœchiométrie, $M$ augmente et semble prendre un continu de valeurs (Fig. 1).

Plusieurs autres exemples d'APP ayant été décrits depuis $\left(\mathrm{Cu}_{3} \mathrm{Au}, \mathrm{Cu}_{3} \mathrm{Pd}, \mathrm{Ag}_{3} \mathrm{Mg}\right)$, deux descriptions ont été proposées pour incorporer ces valeurs non entières dans l'interprétation des données de diffraction $[5,6,7,8]$ :

- Fujiwara [7] considère des empilements réguliers le long de a de domaines d'antiphase, éventuellement de longueurs $M a$ différentes, ce qui conduit à une moyenne pour $M$ pouvant prendre n'importe quelle valeur rationnelle, y compris des valeurs non entières. Dans ces modèles, les frontières de domaines sont toujours rigoureusement planes.

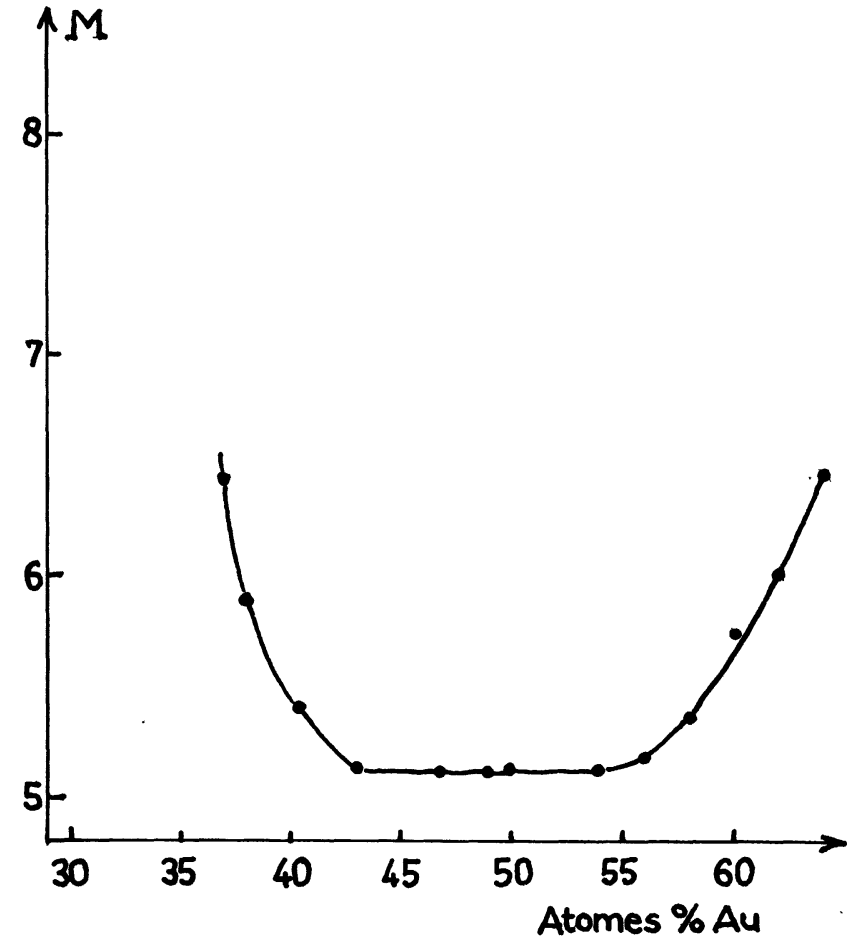

Fig. 1. - Variation de $M$ avec la composition dans $\mathrm{AuCu}$ II d'après Jehanno [5].

[Variation of $M$ versus composition of AuCu II after Jehanno [5].] 
- Jehanno et Pério [5, 6] proposent au contraire un modèle où les valeurs non entières de $M$ proviennent de frontières d'antiphase non planes fluctuant localement autour d'une position moyenne bien définie. Dans ce modèle, qui conduit aussi à des taches de diffraction fines, un continu de valeurs est possible pour $M$.

Nous avons montré par ailleurs [9] que dans l'alliage $\mathrm{Ag}_{3} \mathrm{Mg}$ ordonné, la non-intégralité de $M$ est due à l'empilement parfaitement régulier le long de a de domaines d'extensions différentes (et avec des frontières rigoureusement planes), ce qui conduit pour $M$ aux valeurs rationnelles : $M=\frac{10}{6}(26,5$ at. $\% \mathrm{Mg})$, $M=\frac{7}{4}(25$ at. $\% \mathrm{Mg}), M=\frac{18}{10}(24$ at. $\% \mathrm{Mg}), M=\frac{26}{14}$ $(23,5$ at. $\% \mathrm{Mg}), M=2(22$ at. $\% \mathrm{Mg})$. Cet alliage est une réalisation du modèle de Fujiwara. Nous nous sommes alors tournés vers $\mathrm{AuCu}$ stoechiométrique qui paraissait être le meilleur exemple pour tester l'autre modèle et tenter de mettre en évidence une différence fondamentale d'avec $\mathrm{Ag}_{3} \mathrm{Mg}$.

2. Observations et discussion. - Deux alliages de type $\mathrm{AuCu}$ II ont été préparés (50 at. \% Au et 55 at. \% $\mathrm{Au})$. Après laminage, ils ont subi des traitements thermiques : recuit $24 \mathrm{~h}$ à $600^{\circ} \mathrm{C}$, puis 3 jours à $400^{\circ} \mathrm{C}(50 \%)$ et 3 jours à $370{ }^{\circ} \mathrm{C}(55 \%)$. Puis, amincis électrolytiquement, ils ont été observés au microscope électronique Philips EM300. L'observation d'une seule direction d'antiphase nous a simplifié l'interprétation.

L'échantillon $\mathrm{AuCu}$ II 50 at. \% $\mathrm{Au}$ présente un diagramme de diffraction où, tous les ordres de réflexions étant visibles, on a, sans aucune ambiguïté, exactement $M=5$, ce qui semble confirmer parfaitement la structure Johansson-Linde (Fig. 2). Cepen-

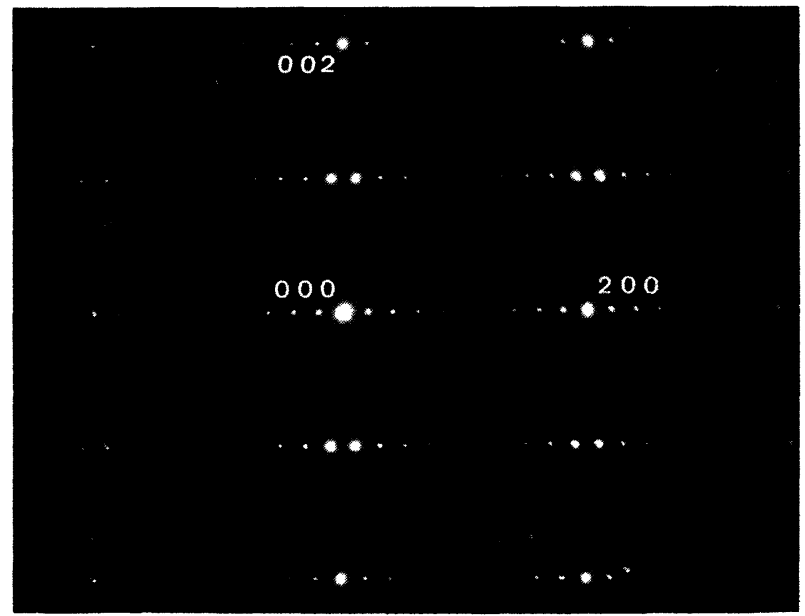

Fig. 2. - Diffractogramme d'un échantillon de $\mathrm{AuCu}$ II à 50 at. \% $\mathrm{Au}$.

[Diffraction pattern of 50 at. \% $\mathrm{Au} \mathrm{AuCu}$ II sample.] dant l'image obtenue avec un diaphragme d'objectif englobant les taches APP donne des franges assez floues et sinueuses (Fig. 3), ce qui tend à prouver que $\mathrm{AuCu}$ II est une véritable APP et non une structure à longue période ordinaire, type $\mathrm{Ag}_{3} \mathrm{Mg}$, caractérisée par des frontières parfaitement rectilignes et fines. Ceci est confirmé par l'examen de l'échantillon à 55 at. $\% \mathrm{Au}$. Sur la figure 4, qui est aussi un plan $\left(\mathbf{a}^{*}, \mathbf{c}^{*}\right)$, deux faits expérimentaux sont notables :

1) On voit clairement le chevauchement des réflexions APP d'indices $\frac{2 n+1}{2 M}, 0, \overline{1}$, avec les réflexions APP d'indices $2-\frac{2 n+1}{2 M}, 0, \overline{1}$. A notre

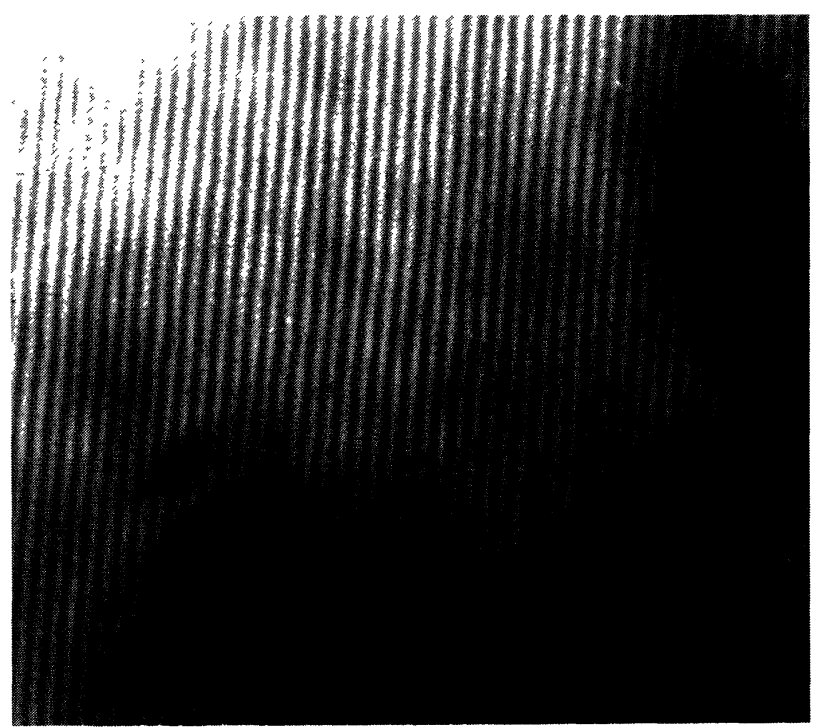

FIG. 3. - Micrographie en fond noir effectuée sur les réflexions APP ( $\mathrm{AuCu}$ II $50 \%$ ).

[Dark field micrograph obtained from PAP reflections ( $\mathrm{AuCu}$ II $50 \%)$.]

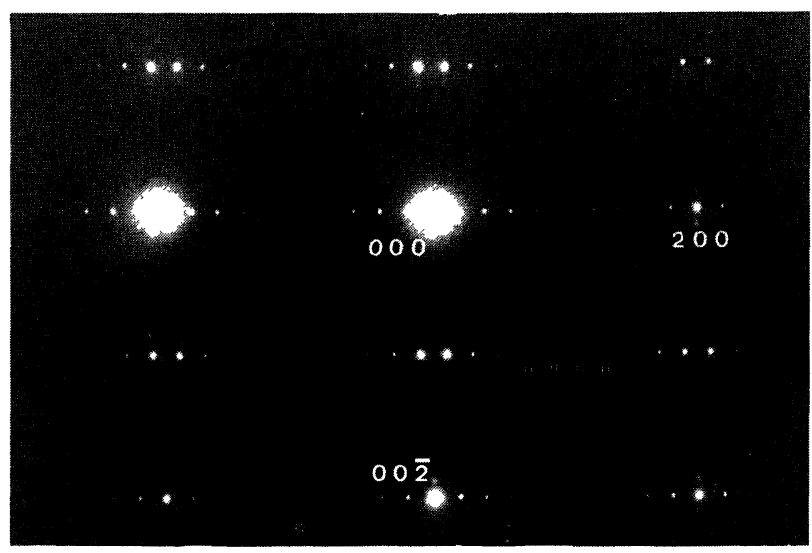

Fig. 4. - Diffractogramme d'un échantillon de $\mathrm{AuCu}$ II à 55 at. \% $\mathrm{Au}$.

[Diffraction pattern of 55 at. \% $\mathrm{Au} \mathrm{AuCu}$ II sample.] 
connaissance, un tel chevauchement n'avait jamais été observé jusqu'ici. Dans le cas de l'échantillon à $50 \%$, les réflexions APP d'ordres supérieurs venaient se superposer exactement sur celles des ordres supérieurs voisins. Ici ce n'est pas le cas, et on mesure : $M=5,12 \pm 0,01$.

2) On remarque également que les rangées réciproques des réflexions APP ne sont pas strictement perpendiculaires à $\mathrm{c}^{*}$, mais sont légèrement inclinées (d'environ $1^{\circ}$ ), ce qui facilite l'observation du chevauchement traduisant l'irrationalité de la période dans l'antiphase périodique proprement dite car chaque tache est, sans aucune ambiguïté, attribuable soit à la série $\frac{2 n+1}{2 M}, 0, \overline{1}$, soit à la série $2-\frac{2 n+1}{2 M}, 0, \overline{1}$. Ce fait correspondrait à des frontières d'antiphase légèrement inclinées par rapport au plan $(b, c)$, et confirme une observation antérieure de Watanabe et Takashima [10].

$\mathrm{AuCu}$ II correspond donc bien au modèle JehannoPério $[5,6]$ qui caractérise une véritable structure APP, selon la définition que nous en proposons [9]. Ceci confirme l'existence dans les alliages de deux types d'ordre à longue période : d'une part les structures infiniment adaptables (ex. $\mathrm{Ag}_{3} \mathrm{Mg}$ ), d'autre part les APP qui sont des structures véritablement irrationnelles (ex. AuCu II).

Outre les satellites des taches fondamentales, qu'on peut attribuer partie à des effets dynamiques et partie à des modulations de position ou de concentration par rapport à la structure moyenne, la figure 4 montre des satellites supplémentaires autour des taches de surstructure et des fondamentales. Observées même à la stœchiométrie, ces taches peuvent être plus ou moins floues et à des positions $k$ par rapport aux taches de surstructure et fondamentales telles que :

$$
k=x a+z c,
$$

$z$ n'étant jamais nul et variant sensiblement entre 0,1 et 0,3 suivant l'individu de macle observé, et $x$ étant encore plus faible, et pouvant, lui, être nul. Bien que l'origine de ces taches reste obscure, il est évident qu'elles sont à relier à une modulation autre qu'une simple modulation d'ordre.

Une étude extensive des valeurs de $M$ en fonction de la composition a été effectuée par Jehanno sur diagramme de poudre [4, 5] (Fig. 1) : cet auteur n'a jamais pu obtenir de valeur inférieure à 5,13 pour la stœchiométrie. La différence entre ces résultats solidement établis et le nôtre pourrait venir d'une différence des traitements thermiques utilisés. Jehanno, pour éviter un grossissement du grain rendant inexploitables les données de rayons $\mathrm{X}$, a recuit systématiquement ses échantillons à $450^{\circ} \mathrm{C}$, alors que les nôtres, recuits à $600^{\circ} \mathrm{C}$, sont ramenés immédiatement à la température de traitement $400^{\circ} \mathrm{C}$. Les échantillons de Jehanno pourraient présenter des fluctuations de composition à moyenne distance (quelques centaines d'angströms) entre des valeurs correspondant aux deux extrémités anguleuses de la courbe de la figure 1 . Dans notre cas, la trempe à partir de $600{ }^{\circ} \mathrm{C}$ éviterait le passage dans la zone des fluctuations (spinodales ?), et les lacunes de trempe assureraient l'homogénéisation ultérieure à courte distance seulement.

Nous remercions le Professeur P. Pério avec qui nous avons eu plusieurs discussions fructueuses.

\section{Bibliographie}

[1] Johansson, C. H. et Linde, J. O., Ann. Phys. 25 (1936) 1.

[2] Ogawa, S. et Watanabe, D., J. Phys. Soc. Japan 9 (1954) 475.

[3] Glossop, A. B. et Pashley, D. W., Proc. R. Soc. A 250 (1959) 132.

[4] Jehanno, G. et Perio, P., J. Physique Radium 23 (1962) 854.

[5] Jehanno, G., Thèse, Univ. d'Orsay, Rapport CEA R2812 (1965).

[6] Jehanno, G. et Perio, P., J. Physique 25 (1964) 966.

[7] Fujiwara, K., J. Phys. Soc. Japan 12 (1957) 7.

[8] Perio, P. et Tournarie, M., Acta Crystallogr. 12 (1959) 1044.

[9] Guymont, M. et Gratias, D., Acta Crystallogr. A, à paraître (1978).

[10] Watanabe, D. et Takashima, K., J. Appl. Cryst. 8 (1975) 598. 